\title{
Mental Communication of Internal Speech with Communicative Associative Robot via Spectral Neurointerface
}

\author{
Evgeniy Bryndin \\ Research Centre "Natural Informatic", Technological Platform Future Medicine, Novosibirsk, Russia
}

\section{Email address:}

bryndin15@yandex.ru

\section{To cite this article:}

Evgeniy Bryndin. Mental Communication of Internal Speech with Communicative Associative Robot via Spectral Neurointerface. American Journal of Applied Psychology. Vol. 10, No. 3, 2021, pp. 55-64. doi: 10.11648/j.ajap.20211003.11

Received: May 15, 2021; Accepted: May 28, 2021; Published: June 3, 2021

\begin{abstract}
The aim of this study is to identify the approach of mental control of robots through the neurointerface. Thought communications with an associative-communicative robot are carried out through the spectral neurointerface of internal speech. Internal speech is an energy physiological process. Internal speech is vibration from the mental vibration of thought. Mental vibration of thought is a process in the mental ethereal field. The vibrations of thoughts are reflected and observed by the mind in the form of semantic sensual images. Vibrations of semantic sensual images generate vibrations of internal speech action (internal speech) in the form of language communicative and associative stereotypes which are perceived by a touch zone of a brain of Wernicke. Internal speech is a linguistic mental vibration, It is felt and becomes internally audible and drawn to attention. The perception of vibrations of internal speech is carried out through energy channels, such as the internal posterior median canal of the spine. The spectral neurointerface perceives these vibrations. Neocortex makes us a reasonable person - allows us to think and talk. The spectral neurointerface is based on the principles of biosensors, bioenergy detectors, spectral analyzers and electrocorticography for neuroimaging parts of the brain that record vibrations of internal speech, such as the lower frontal gyrus, the upper and middle temporal gyrus, the medial prefrontal cortex, the hind parts of the wedge and precline and the dark temporal region, including the posterior Internal speech activity is associated with the semantic memory of the neocortex. As a result of complete electrocortiography, command messages are transmitted to the robot through the spectral neurointerface. Mental control of the robot through the neurointerface makes communication with it natural.
\end{abstract}

Keywords: Spectral Neurointerface, Electrocorticography, Internal Speech, Communicative Robot

\section{Introduction}

Neurointerface is a system for the exchange of information between the human brain and an electronic device. The brain processes incoming sensory stimuli, for example - sound, smell, taste, controls the life and movements of the body. He is also responsible for thinking, memory, emotions and the like. It is noteworthy that these powerful but exquisitely subtle abilities arise from electrical and chemical interactions between the approximately 100 billion cells of which it consists. Each such interaction is reflected in the recorded brain activity, and the neurointerface registers this activity in various regions of the brain and transfers it to external device control commands or vice versa transfers external commands to brain electrical activity. A computer with a specialized program always acts as a translator and simultaneously as an information analyzer in the neurointerface. All brains of each person have common anatomical schemes and synoptic interactions, but the exact sample of connections and interactions varies greatly from person to person, so the program should be able to adjust to the peculiarities of each user's brain. In this case, the program in the computer can process the signal, can be trained, adapting both to the task and to a particular person. For such training, feedback is needed - the program must receive information about whether it correctly interpreted the received signals, whether it "understood" correctly the particular brain with which it works. At the same time, the program can partly manage the patient, orienting him to work with those situations that she recognizes less successfully. We can even say that the brain 
interface - a computer uses artificial intelligence to recognize the types of brain activity.

Neurointerfaces are unidirectional and bidirectional. The former either receive signals from the brain or send them to him. The second ones may send and receive signals simultaneously. Neurointerfaces differ in type:

1. invasive, sensors are placed directly in the cerebral cortex.

2. non-invasive, sensors are placed on the head.

A key feature of the neurointerface is that it allows you to connect to the brain directly. In modern interfaces, brain activity is recorded using electroencephalograms (EEG), magnetoencephalograms (MEG), near infrared spectroscopy (NIRS). MEG allows the measurement of weak magnetic fields generated by ion currents in brain neurons. Superconducting quantum interferometers, or SQUID sensors, are used to detect very weak magnetic fields. Recently, near-infrared spectroscopy (NIRS) has been increasingly used to record brain activity. This is a small device in the form of a hat that is worn on the head. Infrared radiation penetrates through the bones of the skull and adjacent tissues into the frontal and occipital cortex of the brain and allows you to evaluate the oxidation state of hemoglobin, that is, the brain's oxygen consumption. Here, unlike EEG and MEG, a signal of optical nature is recorded absorption of infrared radiation for recording in the motor and prefrontal cortex signals generated by mental score and logical tasks, musical and visual images.

Now the technical problem is measurement accuracy. In non-invasive neurointerfaces - the skull, skin and other layers separating nerve cells from electrodes distort information about the signal. In order to translate electrical activity into understandable commands, the program must be able to: separate the signals it needs and clear them from background noises, adjust to the features of the human brain and translate any information with high accuracy. The brain circuitry is too complex for our analytical and computational capabilities, so at the moment high accuracy is too difficult.

The operation of the interface is based on the analysis of information coming from the patient through four channels. These are the electrical pulses of neurons, their magnetic activity, the flow rate of blood inside the vessels and the change in metabolism.

Many neurointerfaces can be divided into three groups: active, reactive and passive interfaces. The active interface uses changes in brain activity, which is directly and consciously controlled by humans. The reactive interface generates control commands by studying the brain's response to an external signal, such as light or sound. The passive interface analyzes the current activity of the brain, which occurs on its own, in the process of human life. Such interfaces can be useful for creating monitoring systems that monitor the emotional state, detect a decrease in concentration, or a loss of control over the system.

If the neurocomputer interface is connected, for example, with augmented reality glasses, then a person will be able to look at the lighting in the room, switch TV channels and even communicate with a doctor. One of the capabilities of the neurocomputer interface is the transmission of visual information to the brain. Thanks to this, a person can print using a look - however, not very quickly. One of the most famous non-invasive interfaces of this type, it is called R300 speller, allows its owner to dial five characters per minute, the information transfer rate is about 0.5 bits per second. Tianjin University employee Wei Xiwen at the World Robot Conference in China set a record for the speed of typing using neurointerface. Syven's neurointerface spent 0.413 seconds to determine the desired English letter with $100 \%$ accuracy. A group of Chinese and American researchers led by Xiaogan Chen of Tsinghua University have developed a non-invasive neurocomputer interface that allows a person to print a look at a rate of 50 to 60 characters per minute.

An international group of scientists has created a system based on functional spectroscopy in the near infrared region, which allows people with "locked man" syndrome to answer yes or no to a question asked. The Australian company Emotiv develops electronics for neurointerfaces based on EEG. In 2017, American scientists in their study used one of the company's products - the EPOC + helmet, which recognizes waves of electrical brain activity and determines the emotional state of a person.

In 2020, engineers at St. Petersburg Polytechnic University developed Russia's first platform for creating neurotrainers and neurointerfaces, which includes a headset that measures brain activity signals and will allow users to learn how to develop robot control systems using brain signals.

Laboratory of Neurophysiology and Neurointerfaces of Lomonosov Moscow State University. The Neurochat technology is based on the achievements of its employees, which allows people with disabilities to communicate normally with each other and with the world in general. According to the creators, "the headset registers the neurophysiological indicators of the patient and transforms his mental efforts into certain commands for the keyboard of the computer or other actuators. By translating a mental choice of a character into a real set of these characters on the screen, a person letter by letter can type text without the effort of voice and movement. In February of this year, Neurochat was first used in a transcontinental communication session between patients with severe speech and movement disorders. Moreover, each of them "spoke" in his own language, and the neurointerface translated messages to the interlocutor into his native language.

Ideally, neurointerfaces guide the control of the technique by the power of thought. Neurophysiologists and engineers have not yet been able to read thoughts. Not a single device is yet capable of reading human thoughts. The hope that technology will reach such heights is facilitated by the fact that more projects, developments, scientific research in this area, research groups and commercial companies, including large ones that are engaged in the development and development of neurointerfaces, are emerging. There are more than a hundred research groups alone. The largest companies in this area are Neuralink, Mind Technologies, 
Covidien, Compumedics, Natus Medical, Nihon Kohden, Integra Life Sciences, CAS Medical Systems and Advanced Brain Monitoring. Even large firms such as Nissan and Facebook have long announced their intentions to create neurointerfaces.

Elon Musk created Neuralink, with the goal of developing a neurointerface that will allow people to communicate telepathically. In April 2021, Ilona Mask's neurotechnological startup Neuralink published a video with a macaque that plays ping pong on a computer, controlling a virtual racket with the power of thought. Chips were also injected into her brain. According to the developers, using Bluetooth they can be associated with any gadget. First, the monkey was taught to play a video game using a joystick. Her successes were supported by an award in the form of banana puree. At this time, chips recorded macaque brain activity and transmitted data to a computer. There they were processed by a program that learned to recognize the actions of a monkey. After some time, the researchers disconnected the joystick from the computer and watched as the monkey controlled the game with the power of thought, continuing to twist the disconnected controller with his paw. Subsequently, they removed him, and the primate began to play without a single movement of the limbs. Elon Musk said: "We will be able to surpass the power of the human brain by 2030"

Neurointerfaces are used in various spheres of life. The new neuroscience of connecting brains with machines change our lives [1]. Neurointerfaces of interaction [2]. Human brain cloud interface [3]. High resolution passive speech [4]. Technological diagnostics of human condition according to spectral analysis of biofield [5]. Implementation of international telemedicine network with rapid coronavirus registration by resonant technology to neutralize the pandemic [6]. Neuro - processing for the development of neurointerfaces [7]. On-chip tao ${ }_{x}$-based non-volatile resistive memory for in vitro neurointerfaces [8]. Functional and harmonious self-organization of large intellectual agent ensembles with smart hybrid competencies [9].

Neurointerfaces also find use in robot control. The article discusses the control of an associative-communicative robot through the spectral energy interface of internal speech.

\section{Aspects of Thinking}

The process of cognitive thinking of an individual, characterized by a generalized and mediated reflection of reality. Objects and phenomena of reality have properties and relationships that can be learned directly, through sensations and perceptions. The first feature of thinking is its mediated nature. Directly, he learns what the person can't learn directly indirectly, indirectly: some properties through other, unknown entities through known. Thinking always relies on sensations, perceptions, representations and previously acquired theoretical knowledge. Indirect cognition is mediated cognition. The second feature of thinking is its generality. Generalization as a knowledge of general and essential in reality objects is possible because all the properties of these objects are connected to each other. The general exists and manifests itself only in a separate, specific one. The results of cognitive activity of people are recorded in the form of concepts. The concept is a reflection of the essential features of the subject. The concept of a subject arises from research.

Human thinking proceeds in the form of judgments and conclusions. Judgment is a form of thinking that reflects the objects of reality in their connections and relationships. Each judgment is a separate thought of something. The sequential logical connection of several judgments, necessary to solve any mental problem, to understand something, to find the answer to a question, is called reasoning. Reasoning makes practical sense only when it leads to a certain conclusion, conclusion. The conclusion is a conclusion from several judgments, giving us new knowledge about the objects and phenomena of the objective world. Inferences are inductive, deductive and by analogy.

System forms of judgment are analysis and synthesis, comparison, abstraction, concretization, generalization, classification. Thinking acts mainly as a solution to problems, questions, problems that are constantly put forward to people in life. Solving problems should always give a person something new, new knowledge. The search for solutions is sometimes very difficult, so mental activity, as a rule, is an active activity that requires focused attention and patience. The real process of thought is always a process not only cognitive, but also emotionally strong-willed.

For human thinking, the relationship is more significant not with sensual cognition, but with internal speech and language. In a more rigorous sense, internal speech is a language-mediated communication process. If language is an objective, historically established system of codes and the subject of special science - linguistics, then internal speech is a psychological process of formulating and transmitting thought by means of language. Thinking relies on sensations and perceptions. The transition from feeling to thought is a complex process, which consists, first of all, in the isolation and separation of an object or a sign of it, in distraction from a specific, single one and the establishment of a significant common for many objects. Thinking operates with a sense of knowledge. Thinking, as a mental process, models the laws of the surrounding world based on axiomatic positions, establishes connections between objects or phenomena of the surrounding world, reflects the essential properties of objects, which leads to the appearance of ideas about objective reality. Thinking is characterized by the ability of a person to reason, analyze, compare, generalize and draw conclusions.

When an Englishman or an Indian thinks about the same object, the figurative vibration of thought is the same, caused by the object itself or the pronunciation of its name. For this reason, a mind reader whose brain center is in connection with a person's su center can read a hidden figurative. the thought of whose spoken speech he cannot understand. The figurative mental vibration of thought is the same in all people, and its expression as a mental sound vibration is the same in people who speak the same language. If the mental 
language vibrations that encircle thought in the form of speech were the same for all peoples, then the language would be the same.

Consciousness takes a general undifferentiated movement of figurative thought, and continues as a differentiated movement, manifested further in the form of internal speech of the process of thinking. Thinking manifests itself, firstly, as a subject in the subtle form of the mind and its contents, generated by figurative vibrations of thought and, secondly, in the form of linguistic internal speech, as an expression of thought that projects into the world of sensual experience so that they are the source of the impressions of individual experience within it The mental language vibrations of internal speech are the same for people who speak the same language. This allows you to solve the problem of recognizing the internal speech of any specialist through the spectral neurointerface.

\section{Spectral Neurointerface}

Different parts of the brain consisting of neurons are responsible for different physiological functions (Figure 1). Neuronal activity can be traced by electrocorticography.

In the context of neurointerfaces, we will understand them as an external high-tech device connected to our brain and designed to study its abilities, in our case internal speech.

Spectral neurointerface translates brain activity in internal speech into text. Its functioning is based on the work of neural networks with long short-term memory and an open decoder. It is trained and controlled on spectral electrocorticography data obtained from the internal speech of participating professionals. First, the spectral electrocorticogram data obtained when reading the text are taken: the temporal, spatial and frequency characteristics of vibrations are distinguished. The internal speech vibrations are decoded into text using an open deep learning algorithm. The encoder, which recreates the characteristics of the text from the activity of the brain of internal speech, in turn, is based on the work of two bidirectional recurved neural networks with long short-term memory.

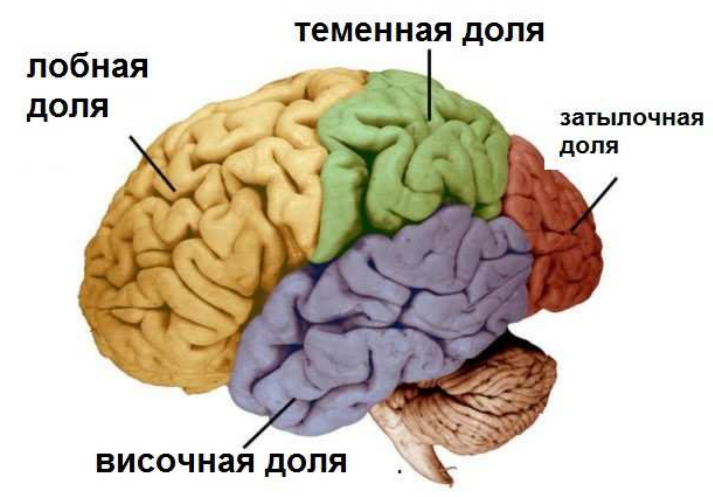

Figure 1. Brain functions.

It is advisable to supply the spectral neurointerface with a regulatory network that allows cleaning the spectral electrocorticogram based on internal speech data of various professionals. This saves the spectral electrocorticogram from artifacts.

Thus, it is possible to achieve high-quality work of the spectral neurointerface in translating the brain activity of internal speech into language speech. At the same time, sufficient performance of the spectral neurointerface is achieved for dialogue in sonorless speech production. Spectral neurointerface can be used for silent people, as well as for telepathic dialogue by internal speech of various specialists.

Spectral neurointerfaces can be widely used for dialogue and control with communicative associative robots over long distances using high-tech wireless communication means for receiving and transmitting messages.

\section{Robot with Communicative Associative Thinking}

Mental communication with a communicative associative robot is carried out through its intelligent communicator agent. A team of professionals with different voices communicate with a communicative associative robot using a spectral energy interface. The communicative robot receives the mental language thoughts of each professional by his internal speech through the spectral energy interface.

The system of communicative associative robot of recognition of internal speech vibrations based on syllable resonators automatically converts syllables into words, and words into messages (Figure 2).

A communicative associative robot perceives words by syllables. It has resonating filters for each syllable and recognizers of syllable morphemes by communicative vibration signals by analogy with the auditory snail. The communication robot has a syllable and punctuation decryptor which sequentially puts text codes of the current syllable or punctuation mark from its memory into the common memory and dampens the resonating filter or recognizer of the previous syllable. The communication robot recognizes syllables and punctuation marks by the decoder and through the neural network structure formats the message, in accordance with the sign markup, and transmits it to the control intelligent system of the robot.

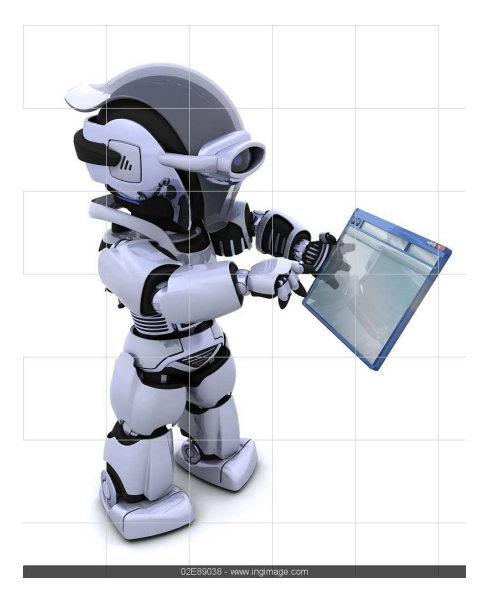

Figure 2. Communicative associative robot. 
The use of robots with communicative associative technological intelligent thinking logic through spectral neurointerfaces is in demand in various spheres of life. Cognitive robots with imitative thinking for digital libraries, banks, universities and smart factories [10].. Robots with artificial intelligence and spectroscopic sight in hi-tech labor market [11]. Robots for communication in public in high-tech industry life and space [12]. System retraining to professional competences of cognitive robots on basis of communicative associative logic of technological thinking [13].. Increase of safety use robots in industry 4.0 by developing sensitivity and professional behavioral skills [14]. Robotization of service with goods and products via automatic cabinet [15].

\section{Communicative Associative Logic of Technological Intellectual Thinking}

Human thoughts are the essence of reason. The mind knows everything in comparison. He compares heard and written thoughts, opinions and worldviews of other people. If your own thoughts, opinions, images and worldviews coincide with other people, then consent comes. If the mind does not agree with the thoughts, opinions, images and worldviews of other people, then the mind forms its own thoughts, opinions, images and worldviews.

Technological intelligent artificial intelligence can compare thoughts, opinions, images and worldviews according to utility criteria. Technological intelligent artificial intelligence can choose thoughts, opinions, images and worldviews according to the criterion of preference. Technological smart artificial intelligence can detect novelty on the principle of opposite (optimal - not optimal; effective not effective; dangerous - safe, etc.) method from nasty based on objective conditions.

The semantic basis of communicative associative logic is an essential dictionary of lexical meanings of representatives of reality.

\subsection{Entity Dictionary}

Let $\mathrm{S}$ be a spelling dictionary, where $\mathrm{S}=\{\mathrm{Si}\}, \mathrm{Si}$ is a morphological word. The word Si refers to the Qij feature of the Mij representative from the Mi set, where $\mathrm{Mi}=\{\mathrm{Mij}\}$. Denote the lexical meaning of the word $\mathrm{Si}$ via $\{\mathrm{Mij}, \mathrm{Qij}, \mathrm{Si}\}$. The relationship of the lexical values of words $\{\mathrm{Si}\}$ with elements of the set $\mathrm{Mi}$ is given by the set of feature relations Qi, where Qi $=\{$ Qij, (Mi, Mij) $\}$.

A set of lexical values associated with a set of characteristic relations with representatives is an essential dictionary. Words in the dictionary are supplied with characteristic indexes according to their characteristic relationships with representatives. The entity dictionary captures the characteristic entities of representatives. The dictionary helps to use words with its lexical meaning and distinguish between representatives whom they call on a symbolic level.

Words are used based on characteristic indices. Each feature has three indexes. One index indicates the subject area of knowledge, the second indicates the situation, the third indicates the situational moment. Words with multiple lexical meanings have multiple sets of indexes. For example, torment (muka) and muka (flour). The word field is used in various subject areas. Each set of indexes defines a lexical word value.

\subsection{Information Needs Technology}

Tasks, problems, tasks and goals are formed in the form of information needs. The text of the information requirement is based on the functional natural language from the elements of the essential dictionary. Functional natural language describes communicative and associative relationships between knowledge elements. Communicative and associative language relationships between knowledge elements follow the relationships of objects, actions, qualities, properties, time, space, etc., which we denote knowledge elements.

The language links the structural, functional and semantic attributes of knowledge elements. The use of words as part of information demand knowledge elements is defined by communicative links. The language has grammatical rules for the speciation of word forms for the creation and expansion of families and the creation of communities, communicative rules for the formation of phrases, sentences of judgments. Each rule has its own definition area. For example, in a deductive generalization rule, the domain of the definition is deductive sets. Deductive rules allow you to organize language words into generalized sets. The generalized set is represented by a convergent feature. The private values of the convergent characteristic are its specificity. In the language, the emphasis in the word, in the sentence, in the judgment between sentences is fixed by synthactics. The impact words in the sentence are shown in bold. For example, I was in China or I was in China or I was in China. Impact syllables are shown in bold. Communicative relationships of content sentences have syntactic notations.

According to the act, ascending parsing and morphological analysis identifies sentences of information need that correspond to acts of thinking and establishes grammatical types and characteristics for each word according to a dictionary of semantic orientation, builds a tree of syntactic subordination of words, determines the communicative relationship of words, phrases and sentences. For analysis, you use an essential dictionary, networks of phrases, sentences, judgments, information families, and communities.

Parsing is carried out by language syntax markup of information demand and its structural situationalcharacteristic markup. By parsing, the grammatical values of knowledge elements are determined, words in sentences, phrases in sentences, sentences in judgments, elementary judgments in composite judgments are distinguished, grammatical types of words, species groups of phrases according to grammatical types of words of basics and additional, grammatical types of lexical values of 
information niches of sentences, and species relationships of sentences of elementary judgments are identified.

Morphological analysis is carried out according to linguistic morphological markup and schematic situationalcharacteristic. In the process of morphological analysis, morphological values of knowledge elements and situationalcharacteristic schemes of morphological values are determined.

The selection of sentences is carried out according to a syntactic structure, in which words are assigned features and indexes of representatives, grammatical types and lexical values, semantic values to phrases. The information niches of the quotation are determined by the act-based upstream parsing and morphological analysis of the information demand texts. The resulting text, after an act analysis, enters the system of symbolic and linguistic implementation of information needs - a system of imitation of thinking.

The input information of the system is the information demand and attributes obtained in the process of syntactic and morphological analysis. According to information obtained as a result of syntactic and morphological analysis of information demand, the system of imitation of thinking carries out its semantic analysis.

The information requirement type is first recognized. The information need may be questionable or prompting. Semantic analysis then determines whether there is an implementation of the knowledge base information requirement. If it is not implemented, it is determined whether the information requirement is a typical baseline. If the information requirement is not a generic basic requirement, the analysis determines whether it consists of a generic basic information requirement. If it does not consist of generic basic information needs, semantic analysis determines whether there is a necessary and sufficient set of implementation procedures in the knowledge base and a necessary and sufficient set of knowledge elements in the knowledge base to implement it.

If, as a result of analysis, it turns out that in the knowledge base there are elements for realizing the information need, then the simulation system of thinking forms the implementation of the information need from the knowledge elements of the information need and the knowledge elements for implementation from the knowledge base. By marking the text of the information demand, the system determines pointers to the necessary sets of knowledge elements in the knowledge base in the subject area specified by the user, and to the implementation procedures in the skill base, and determines the implementation scheme according to the type and layout of the need.

The markup indicates the attributes of knowledge elements: schematic, characteristic, grammatical (syntactic, morphological), semantic and lexical.

Pointers to knowledge and implementation elements are formed through an information receptive field. The information receptive field contains information connections with inputs of a network of phrases, sentences, judgments, as well as with implementations of typical basic information needs. It contains instructions on the necessary and sufficient set of implementation procedures for each lexical value of the information action and on the necessary and sufficient set of knowledge elements for each implementation procedure, as well as information links with implementation procedures stored in the skills base.

Markup of information demand and user-defined area of knowledge is used to define pointers to find necessary and sufficient set of implementation elements in the knowledge base, necessary and sufficient set of knowledge elements in the knowledge base, schemes and structures of formation of new phrases, sentences, judgments.

The system of simulation of thinking starts the procedure of building a network of element-by-element realization of information demand from the necessary and sufficient set of knowledge elements and implementation procedures. It builds a network of element-by-element implementation of information needs in databases of knowledge and skills. It then performs initial markup of knowledge elements and implementation procedures and their relationships. Markup defines the characteristic participation of words in phrases, the situational-characteristic participation of phrases in sentences and the situational participation of sentences in judgments.

After marking the element-by-element implementation network, it is launched to realize the information demand.

Information needs are considered feasible if the syntax structure of the implementation, lexical and semantic values of the components, as well as implementation procedures are determined.

If the input information requirement is a combination of typical information requirements or a valid modification thereof, it is realizable.

A typical incentive information requirement consists of an information action and an information condition. If the information need is prompted, then it explicitly indicates the action.

If the information requirement or the information requirement realization contains an information action, then the standard realization procedure is started. Reference to implementation procedure is indicated in information action markup.

For example, let the imperative information need be given "to find the sum of $\mathrm{X}$ plus $\mathrm{Y}$, with $\mathrm{X}$ equal to 15 , Y equal to 10 (Figure 3 ). $\mathrm{X}$ is $15, \mathrm{Y}$ is 10 is an information condition. The communicative phrases "X equals" and "equals 15" in the information condition of the information action "plus" initiates the replacement of $\mathrm{X}$ with 15. According to the information condition "X equals 15 , Y equals 10 ," X will be replaced by 15 , and $\mathrm{Y}$ by 10 in the typical implementation of the information demand. The lexical value of the informational action "plus" in the essential symbolic language dictionary contains a reference to the analytical sign "+" in the analytical entity dictionary. The sign "+" in the analytical entity dictionary refers to the typical procedure for adding two arguments. 


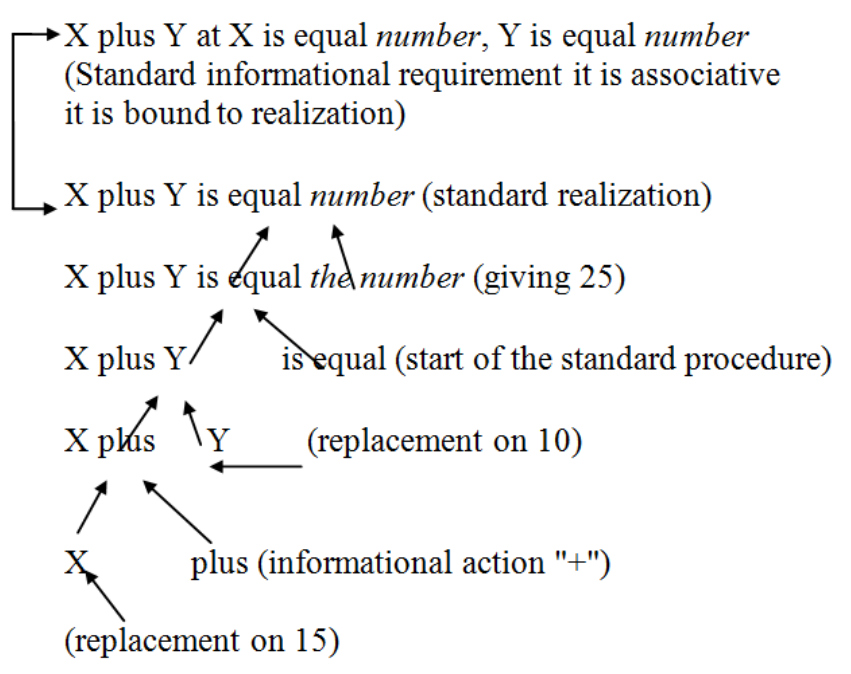

Figure 3. Network structure of addition.

The question information need is a question proposal. If the information need is questionable, it explicitly indicates the question phrase defining the direction of implementation.

The implementation of the question information need is built either in the network of proposals or in the network of judgments. In networks, the semantic value of the realization of the need is revealed, either in the form of a proposal or judgment.

After implementation, the new information need is entered into the knowledge base and linked to the knowledge element, which is its implementation.

The technology of implementing the communicativeassociative logic of imitation thinking allows you to develop interactive educational systems of a natural language level in various subjects in the educational space. The subject area of knowledge is presented in the form of a communicativeassociative network of information needs and their implementations, consisting of elements of knowledge. Communicative phrases form sentences, communicative sentences form judgments. The association between the information need and its implementation is associative.

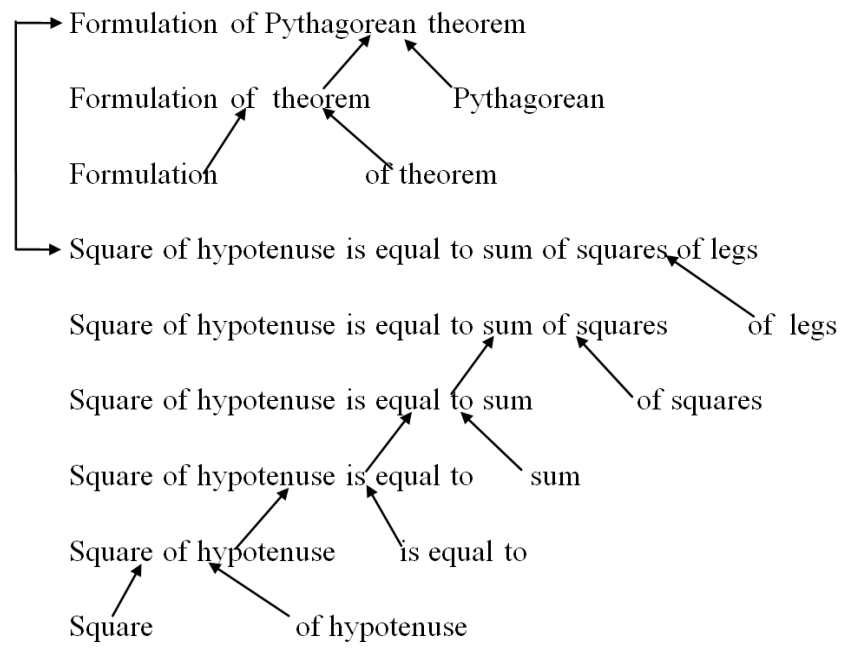

Figure 4. Associative communicative network of Pythagorean theorem.
For example, let the imperative information need "Formulate Pythagorean theorem" be given (Figure 4). By the lexical meaning of the word "formulation," we find through the essential dictionary the representative phrase "formulation of the theorem" in the network of the subject area of knowledge indicated in the markup of information need. Then, through the tree of communicative phrases with lexical values of information demand, by synthesizing them, we get to the associative vertex "the formulation of the Pythagorean theorem. Further, along the associative connection from the associative vertex of the information need, we move to the associative vertex of the implementation "the square of the hypotenuse is equal to the sum of the squares of the catets.

The realization of an information requirement can itself be an information requirement. In this case, by the communicative tree of the lexical values of the theorem, we get to the associative vertex by synthesizing them. Then, by associative connection, we determine the implementation of the "Formulation of Pythagorean theorem"

The words of information demand have markup indicating either a lexical value, or a computational procedure, or a behavioral procedure. For example, the word "add" can be a lexical value, either an arithmetic action or a behavioral action (add cubes). For judgments, the subject area and situation are indicated. For quotations, you specify the situational moment and the characteristics of the lexical values. The reflection of the semantics of subject knowledge is carried out using a symbolic-linguistic sign system based on communicative-associative logic and subject-situationalcharacteristic relations of entities of reality and abstraction objects with knowledge elements of the essential dictionary.

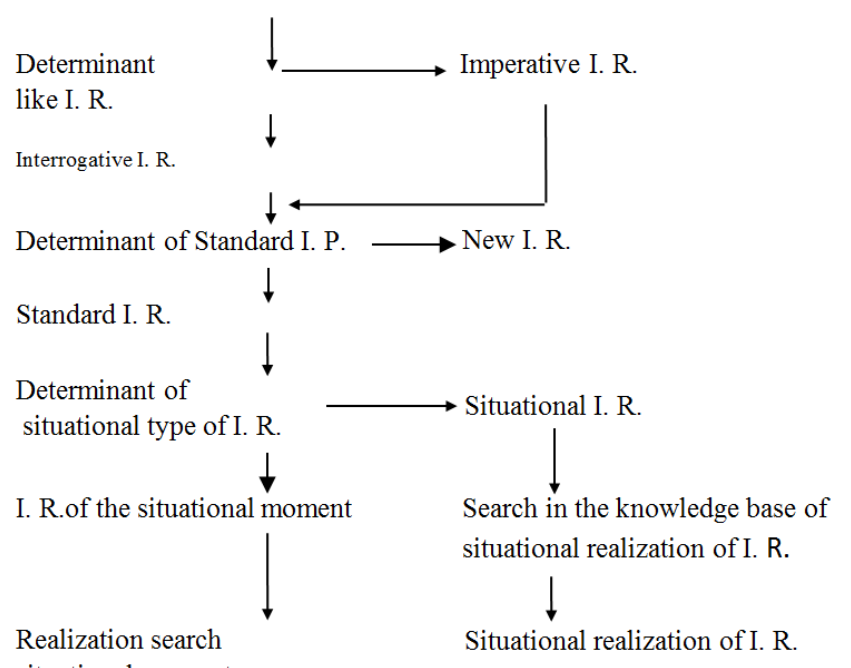

situational moment

in the knowledge base

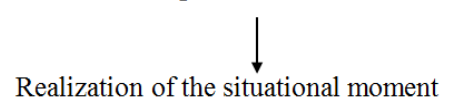

Figure 5. Realization of standard information requirement (I. R.).

The information demand implementation system uses a knowledge base and a skill base. The implementation of the information demand is taken either from the knowledge base, or is developed by a typical procedure for implementing the 
skills base according to the current information demand, or a network of element-by-element implementation according to the combined information demand is formed. Schemes for implementing information needs are obtained from a study of educational practices in the formation of imitative thinking. The diagrams are shown in Figures 5-6.

After implementation, the new information need is entered into the knowledge base and associated with the knowledge element, which is its implementation. The variation of such information needs is carried out in a variable part, which are implemented by standard procedures common to them.

The realization of combined information needs is developed by the investment, variation and combination of implementations of information needs, which are located in the knowledge base of the system. New information needs are built either by sequentially merging existing needs from implementations, by investing one in another, or by merging and investing at the same time.

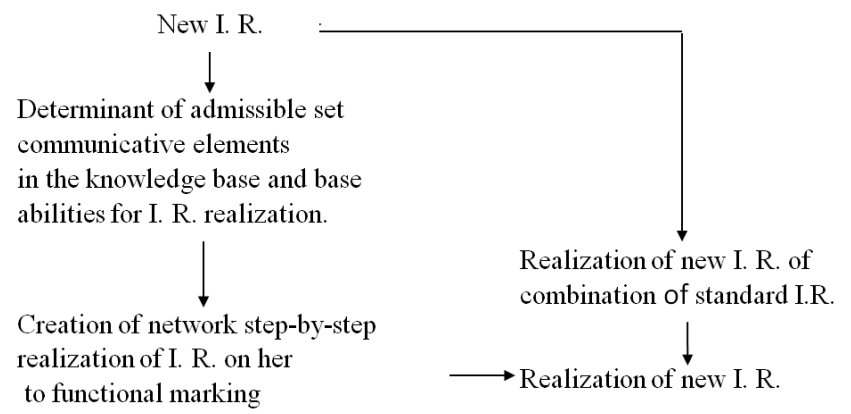

Figure 6. Realization of new information requirement (I. R.).

Retraining system is used to work with the new concepts (Figure 7).

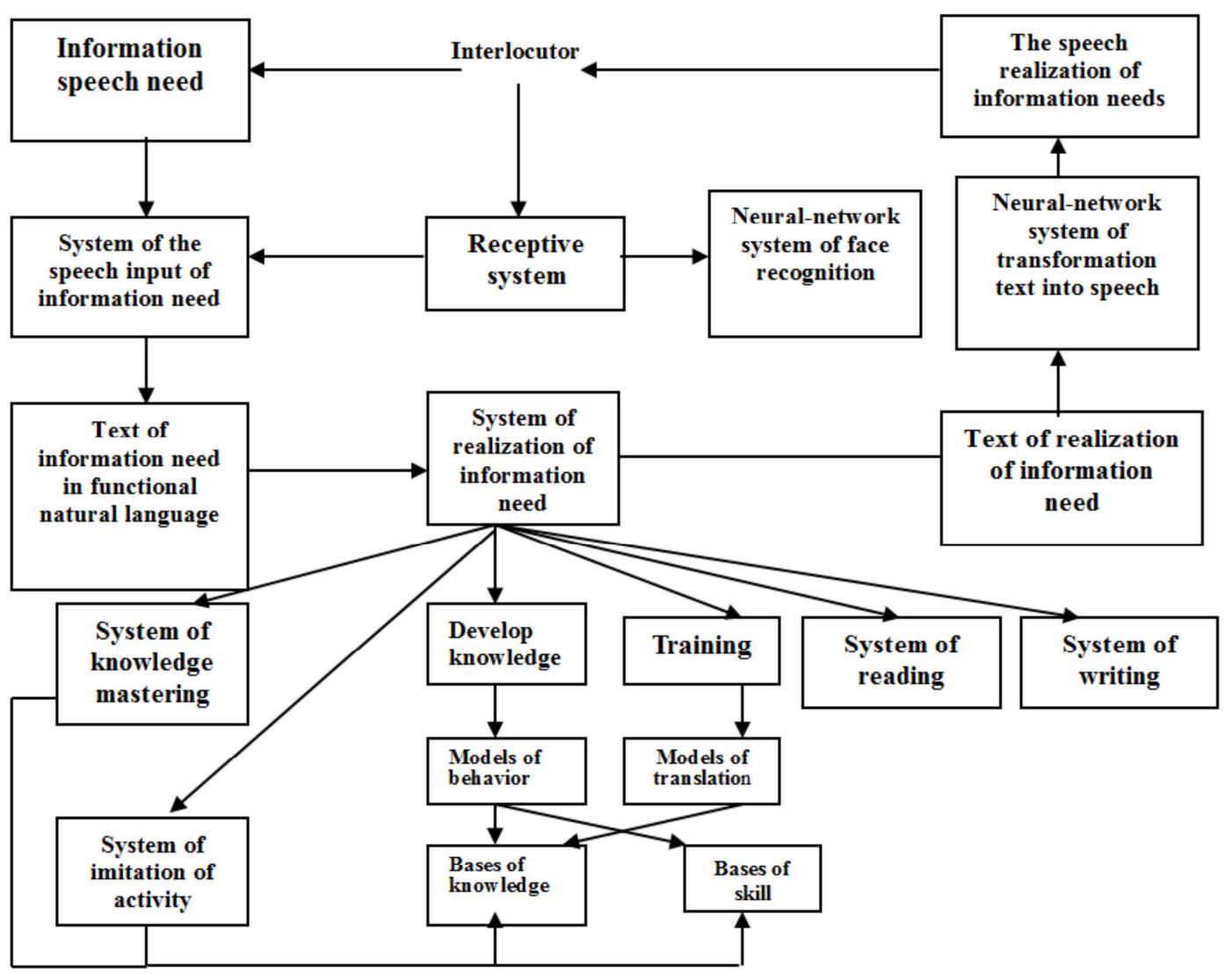

Figure 7. Retraining system.

\section{Connection of Neurointerface to Robot}

With the development of wireless data transfer technologies, the neurointerface makes the connection of the neurointerface with the robot flexible. Wireless interfaces and communication protocols for use as a primary means of communicating a modern neurointerface with a robot can be ZigBee. Protocols are regulated by the IEEE 802.15.4 standard. ZigBee and IEEE 802.15.4 describe wireless personal area networks (WPAN). The ZigBee specification focuses on applications that require guaranteed secure data transmission at relatively low speeds and the possibility of long-term operation of network devices from autonomous power supplies. Due to the fact that ZigBee technology allows you to build not only simple network topologies (point-to-point, tree and star), but also a self-organizing and self-restoring mesh topology with relay and message routing, the protocol is well suited for building branched, scalable networks consisting of dozens of neurointerfaces, for example, it can be a military unit each combat unit of which is equipped with an individual neurointerface.

Advantages of ZigBee: flexibility in self-organization and 
self-healing; Simple network deployment techniques high level of resistance to electromagnetic interference; high security; Lack of frequency licensing; low power consumption.

But ZigBee lack of standardization and lack of a single hardware and software platform for developing complex applications and high-level debugging boards is a disadvantage.

In the case of using the neurointerface in a social environment, it may be useful to use the wireless $\mathrm{Z}$-wave communication protocol. The protocol is initially focused on application in home automation systems, where neurointerface applications are already being found. Z-Wave is promoted by a consortium of developers in the form of functionally completed electronic modules designed to be embedded in various electronic products. At the same time, the technology uses frequencies up to $1 \mathrm{GHz}$ for its operation and is mainly intended for transmitting control commands. Thus, Z-wave technology is suitable for controlling the neurointerface, but not for full data transfer.

Existing wireless radio systems (Wireless RF) can be used to solve the problem of communicating a low-speed neurointerface with a computer. These systems use licensefree frequencies as a rule in the $433 \mathrm{MHz}$ range and have a communication range of up to $100 \mathrm{~m}$ while providing a data transfer rate of up to $115 \mathrm{kbit} / \mathrm{sec}$. Simple wireless radio systems can use a symmetric block encryption algorithm with an encoding system.

The most suitable technology for implementing the wireless neurointerface is the Bluetooth Low Energy wireless data technology, best known by its abbreviation (BLE). A special advantage of the technology is its prevalence on a huge number of devices, primarily devices with autonomous power supply, i.e. mobile or wearable. The BLE technology is specifically designed for short-range wireless communication. This fact underlines the high energy efficiency of BLE, which is extremely important when implementing the neurointerface with autonomous power supply. Of the advantages of BLE technology, the following should be noted: a high level of implementation in existing mobile devices; Worldwide high data transfer rates, including encryption; high reliability and resistance to electromagnetic interference. It should be noted the problems with the identification of devices in the network and the low communication range in urban development. The latter in the case of the use of BLE in the neurointerface is not significant, since the neurointerface is usually operated in the immediate vicinity of the robot.

Modern wireless data technologies can be used in communication of a neurointerface with a robot for wide purposes over short distances.

\section{Conclusion}

On the way to creating an ideal neurointerface that can convey and translate thoughts with high accuracy between the brain and computer, and will also have many areas of use and huge distribution among the population, there are several obstacles. Basically, these obstacles represent a number of modern technical, ethical and legal restrictions. Already, large corporations own our personal information, and with the development of neurointerfaces they will be able to read our thoughts, few will like it, but this is not the worst. The scary thing is that using the brain-computer interface, if necessary, it will be possible to change a person's personality or completely intercept control over his body. No matter how high the hacking protection is. Neurointerfaces will be susceptible to computer viruses and attackers. For this reason, developers will have to provide an appropriate level of security.

Neurointerfaces are progress, not stop it. Attracting intellectual resources greatly contributes to the development of technology, and once again convinces that neurointerfaces are approaching at incredible speed and people should be ready to meet them.

The use of robots with communicative associative technological intelligent thinking logic through spectral neurointerfaces is already in demand in various spheres of life. Thought control of robots over long distances requires interdisciplinary research in the field of information transmission through communication means. This will expand the use of robots in dangerous conditions and even in space.

\section{References}

[1] Nicolelis M. A. (2011). Beyond Boundaries: The New Neuroscience of Connecting Brains with Machines - and How It Will Change Our Lives, 354 pp. New York, Times Books.

[2] Zander T. O. Team Phy PA: Brain-computer interfacing for everyday human-computer interaction. Professional network for scientists and researchers "ResearchGate», 2017. 61 (2). pp. 209-216.

[3] Lebedev M. Human Brain/Cloud Interface. Frontiers in Neuroscience. 2019. Vol. 13. P. 1-24.

[4] Sinkin M. V., Osadchiy A. E., Lebedev M. A. High resolution passive speech mapping in dominant hemisphere glioma neurosurgery. Russian Journal of Neurosurgery 2019; 21 (3).

[5] Evgeniy Bryndin, Irina Bryndina. Technological Diagnostics of Human Condition According to Spectral Analysis of Biofield. Advances in Bioscience and Bioengineering. Volume 7, Issue 3, 2019. Pages: 64-68.

[6] Evgeniy Bryndin. Implementation of International Telemedicine network with Rapid Coronavirus Registration by Resonant Technology to Neutralize the Pandemic. Computational Biology and Bioinformatics. Vol. 8, Issue 2, 2020, pp. 29-35.

[7] N. I. Chervyakov, Pavel Lyakhov, Maria Kiladze. EEG Neuro - processing for the development of neurointerfaces. IOP Conference Series Materials Science and Engineering. July 2020.

[8] Maksim Zhuk and ather. On-Chip $\mathrm{TaO}_{x}$-Based Non-volatile Resistive Memory for in vitro Neurointerfaces. Front. Neurosci., 26, February 2020. 
[9] Evgeny Bryndin. Functional and Harmonious SelfOrganization of Large Intellectual Agent Ensembles with Smart Hybrid Competencies. American Journal of Software Engineering and Applications. Vol. 9, Issue 3, 2021.

[10] Evgeniy Bryndin. Cognitive Robots with Imitative Thinking for Digital Libraries, Banks, Universities and Smart Factories. International Journal of Management and Fuzzy Systems. V. 3, N. 5, 2017, pp 57-66.

[11] Evgeniy Bryndin. Robots with Artificial Intelligence and Spectroscopic Sight in Hi-Tech Labor Market. International Journal of Systems Science and Applied Mathematic, V. 4, № 3, 2019. Pages: 31-37.

[12] Evgeniy Bryndin. Robots for Communication in Public in High-
Tech Industry Life and Space. Frontiers Journal of Current Engineering Research. Volume 1, Issue 1, 2019. P. 1-10.

[13] Evgeniy Bryndin. System retraining to professional competences of cognitive robots on basis of communicative associative logic of technological thinking. International Robotics Automation Journal. 2019; 5 (3): 112-119.

[14] Evgeniy Bryndin. Increase of Safety Use Robots in Industry 4.0 by Developing Sensitivity and Professional Behavioral Skills. American Journal of Mechanical and Industrial Engineering. Volume 5, Issue 1, 2020. Pages: 6-14.

[15] Evgeny Bryndin. Robotization of Service with Goods and Products via Automatic Cabinet. International Robotics \& Automation Journal, Volume 7, Issue 1, 2021. pp. 20-22. 